\title{
Relationships of First Trimester Body Mass Index and Weight Change with Persistent Organic Pollutants Concentrations in Canadian Pregnant Women
}

\section{Marianne Levesque}

Ottawa Hospital Research Institute

Mariame 0. Ouedraogo

Ottawa Hospital Research Institute

Romina Fakhraei

Ottawa Hospital Research Institute

Alysha L.J. Dingwall Harvey

Ottawa Hospital Research Institute

Elizabeth Bratton

University of Ottawa Faculty of Medicine

Mark C. Walker

Ottawa Hospital Research Institute

\section{Linda Dodds}

Dalhousie University Faculty of Medicine

Laura M. Gaudet ( $\triangle$ laura.gaudet@kingstonhsc.ca)

https://orcid.org/0000-0002-7527-5354

\section{Research}

Keywords: Maternal Obesity, Early Gestational Weight Changes, Persistent Organic Pollutants, Maternal and Fetal Health

Posted Date: November 9th, 2021

DOI: https://doi.org/10.21203/rs.3.rs-1032665/v1

License: (c) (1) This work is licensed under a Creative Commons Attribution 4.0 International License.

Read Full License 


\section{Abstract \\ Background}

Persistent organic pollutants (POPs) are toxic chemicals with demonstrable effects on pregnancy and neonatal outcomes. The associations of early pregnancy body mass index (BMI) and antenatal weight changes with circulating POP concentrations are poorly understood in the Canadian context. The objective of this study is thus to examine the relationships between first trimester maternal BMI, weight change from pre-pregnancy to 6-13 weeks of pregnancy (early gestational weight change), and first trimester plasma POP concentrations among Canadian pregnant women.

\section{Methods}

We conducted a secondary analysis of data collected as part of the Maternal-Infant Research on Environmental Chemicals (MIREC) Study. We determined the POP concentrations across first trimester BMI category (underweight/normal weight, overweight, class I \& II obese, and class III obese) and early gestational weight change categories (weight loss, weight neutral/gain) and tested for overall differences using Kruskal-Wallis tests. Associations between first trimester maternal BMI and early gestational weight changes with the plasma concentrations of 41 POPs measured in the first trimester were further evaluated using unadjusted and adjusted censored regression models.

\section{Results}

Eleven of 41 POPs were detectable in at least $50 \%$ of the MIREC participants and could be analyzed for their relationships with first trimester BMI and weight change. The majority of POPs were inversely associated with first trimester BMI after controlling for the main confounding variables. Although not statistically significant, POP plasma concentrations tended to be generally higher in participants who lost weight compared to those who gained weight or whose weight stayed relatively stable from prepregnancy into the first trimester.

\section{Conclusions}

Our findings lend support to the hypothesis that pregnant women with obesity may have higher bioaccumulation of POPs within their adipose tissues than normal weight pregnant women. Additionally, early gestational weight loss appears to be associated with the highest circulating POP levels. Future studies should focus on the effect of weight changes on POPs concentrations across trimesters.

\section{Introduction}


Maternal obesity is a major health concern in pregnancy. Mothers affected by this condition are at significantly higher risk of miscarriage, gestational diabetes, gestational hypertension, pre-eclampsia, thromboembolic events, and stillbirth compared to their normal-weight counterparts[1, 2]. Children born to mothers with obesity are at increased risk of congenital anomalies, and of developing obesity later in life [3]. Gestational weight gain (GWG) - irrespective of pre-pregnancy weight class - may affect pregnancy outcomes. Excessive or inadequate GWG has been associated with detrimental effects on maternal and fetal outcomes[4]. Health organisations across the world have established healthy GWG recommendations based on pre-pregnancy maternal body mass index (BMI) in attempts to mitigate pregnancy risks. For women with obesity $\left(\mathrm{BMI} \geq 30 \mathrm{~kg} / \mathrm{m}^{2}\right)$, Canadian recommendations include: preconception weight loss whenever possible, and a total GWG of 5.0 to $9.0 \mathrm{~kg}[5]$. In contrast, normal-weight women (BMI $18.5-24.9 \mathrm{~kg} / \mathrm{m}^{2}$ ) in Canada are recommended to gain between 11.5 to $16 \mathrm{~kg}$ during a pregnancy[6]. GWG was reported to be inadequate in $15.8 \%$ and excessive in $57.9 \%$ of patients in a small Canadian cohort study[7].

Based on current evidence, weight changes in non-pregnant individuals are suspected to promote mobilization of lipophilic environmental toxins referred to as persistent organic pollutants (POPs) [8-10]. POPs have recently become a subject of interest given their characteristics and adverse health effects. POPs are typically polyhalogenated organic compounds that are highly lipid soluble. This enables them to easily accumulate and biomagnify along food chains[11].They are extremely resistant to degradation, and easily dispersed across wide geographical areas[11]. They can travel through soil, water, and air[11]. Most POPs have been released into the environment via agricultural and pest/disease vector control products multiple decades ago[12]. They remain present in the environment today despite having been banned from use in most countries[12]. Human exposure may occur through inhalation, dermal absorption, and dietary consumption of fatty fish, meat, and dairy products[12-14].

POPs are measurable in the tissues of pregnant women and their offspring[15-19] with demonstrable effects on pregnancy and long-term pediatric outcomes[20,21]. Neonates are easily susceptible to the toxic effects of environmental pollutants due to their unique body composition, immature organ systems, limited metabolism and elimination processes and rapid development[22].

Because of the inherent characteristics of POPs, it has been hypothesized that weight stability, particularly in overweight or obese individuals who are likely to have a greater body burden of POPs, is protective against the release of toxic lipophilic chemicals through their sequestration in the adipose compartment[10]. Evaluation of POP burden relative to weight change in pregnancy has thus far been limited, particularly in North America. In 2016, Fisher and colleagues reported significantly higher concentrations of plasma POPs in women with a normal pre-pregnancy BMI compared to women with higher BMIs participating in a large pregnancy cohort [23]. The relationship with first trimester BMI and weight fluctuations was not considered by Fisher and colleagues. For this reason, we aimed to assess the association between gestational weight change in early pregnancy with maternal POP plasma concentrations using data collected in the Maternal-Infant Research on Environmental Chemicals (MIREC) study. We hypothesised there would be an increase in plasma levels of POPs in women 
experiencing weight loss in early pregnancy, particularly in those with a $\mathrm{BMI} \geq 30 \mathrm{~kg} / \mathrm{m}^{2}$, based on previously described POPs' metabolism.

\section{Methods}

\section{Study population}

Our study cohort was assembled using data collected from the MIREC Study, a multisite Canadian prospective cohort study that aimed to examine potential adverse health effects of prenatal exposure to environmental chemicals on pregnancy and infant health. Specific details regarding the MIREC study have been previously described[24] and are briefly indicated here. Between 2008 and 2011, a total of 2,001 women were recruited from 10 cities across Canada during their first trimester of pregnancy (6-13 completed gestational weeks) and followed through delivery. Study exclusion criteria included: an inability to communicate in English or French, less than 18 years of age, above 14 weeks gestation at the time of recruitment, the presence of a known fetal anomaly in current pregnancy, or a history of maternal medical complications. The MIREC database contains information collected at study visits during pregnancy and post-delivery. In addition to the MIREC questionnaire information, this database is supplemented by maternal and infant data collected through medical chart extraction. For the present study, the population was restricted to MIREC mothers with measured first trimester POP concentrations data.

\section{Study variables}

The outcome variables of interest in this study were POP plasma concentrations. Data on 41 different POPs measured from first trimester maternal blood plasma were used. These included 24 polychlorinated biphenyls (PCB 28, 66, 74, 99, 101, 105, 118, 128, 138, 146, 153, 156, 163, 167, 170, 178, 180, 183, 187, 194, 201, 203 and 206 and Aroclor 1260), 10 organochlorinated pesticides (beta-hexachlorocyclohexane [ $\beta-\mathrm{HCH}]$, hexachlorobenzene, cis-nonachlor, trans-nonachlor, oxychlordane, dichlorodiphenyldichloroethylene [DDE], p,p'-dichlorodiphenyltrichloroethane [DDT], Mirex, Parlar 26 and 50), 6 polybrominated diphenyl ethers (PBDE 28, 33, 47, 99, 100, 153) and hexabromobiphenyl (PBB 153). The POP concentrations were measured by the Centre de Toxicologie du Québec of the Institut National de Santé Publique du Québec. Laboratory methods and calculations for the POP concentrations have been previously described[23]. We considered for analysis all POPs measured in first trimester maternal plasma.

The main explanatory variables were first trimester $\mathrm{BMI}$ and early weight change during pregnancy. First trimester BMI was determined from maternal height and weight measurements taken during the first MIREC visit, which took place between weeks 6 and 13 of pregnancy. Participants' BMI was categorized into underweight $\left(<18.5 \mathrm{~kg} / \mathrm{m}^{2}\right)$, normal weight $\left(18.5-24.9 \mathrm{~kg} / \mathrm{m}^{2}\right)$, overweight $\left(25.0-29.9 \mathrm{~kg} / \mathrm{m}^{2}\right)$, obese class I \& II (30.0-39.9 kg/m²), and obese class III ( $\left.\geq 40.0 \mathrm{~kg} / \mathrm{m}^{2}\right)$. We estimated early pregnancy weight changes as the percentage change in weight recorded at the time of the first MIREC visit from the self- 
report pre-pregnancy weight. First trimester weight change was dichotomized into first trimester "weight loss" (if the participant had a weight loss of $\geq 2 \%$ of their total body weight early in pregnancy) and "weight neutral or gain" (if the participant had a weight loss of $<2 \%$ of their total body weight or any weight gain reported early in pregnancy). We could not find a percent weight change cut-off for pregnant women in their first trimester in the literature. We, therefore, relied on clinical expertise and relevance to set the cut-off of $2 \%$.

Several confounders were used to adjust for the relationship between the two explanatory variables and POP concentrations. The selection of confounders was based on a thorough review of variables considered in similar studies $[15,23,25]$ and directed acyclic graphs[26]. We considered gestational age at the time of the first MIREC visit (ranging between 6 and 13 weeks), parity $(0,1,2,3+$ ), maternal age $(<25,25-29,30-34,35+$ years), maternal education (high school or less, some college or college degree, undergraduate degree or higher), maternal smoking status (current (including women who quit smoking during pregnancy), former, never), and women's place of birth (Canada, other). Self-reported prepregnancy BMI (underweight, normal weight, overweight, obese) was included as a confounder of the association of weight change with POPs.

\section{Statistical Analysis}

All statistical analyses were conducted using SAS/STAT® software, version 9.4 of the SAS system (SAS Institute, Cary, NC, USA). Characteristics of the study participants are presented as frequencies and percentages for categorical variables and means and standard deviations for continuous variables. Sample plasma concentrations were below the laboratory level of detection (LOD) for the majority of POPs (30 out of the 41). As a result, censoring techniques were used to generate descriptive statistics. All POPs were expressed in microgram per kilogram serum lipid, as done previously[23]. The concentration range was determined for each POP where possible. The geometric means with corresponding 95\% confidence intervals were obtained using Maximum Likelihood Estimate (MLE). Additionally, KaplanMeier (KM) methods were used to determine medians with 95\% confidence intervals. Subsequent analyses were only performed on the 11 POPs with concentrations above the LOD for at least $50 \%$ of the sample.

For the eleven POPs with less than 50\% censored data, we estimated geometric means and $95 \%$ confidence limits of lipid-adjusted POP concentrations by first trimester BMI category. As there were too few underweight women $(\mathrm{N}=25)$ to be analyzed separately, they were grouped with the normal weight category. The relationships between first trimester BMI and first trimester plasma lipid-adjusted POP concentrations were assessed using Kruskal-Wallis tests, as the assumption of normality of POP concentrations did not hold. A significance level of $5 \%$ was considered for the overall difference in lipidadjusted POP concentrations among first trimester BMI categories.

Censored regression models were built assuming a lognormal distribution for the POP concentrations. Censored regressions are the preferred approach to analyze datasets with an important number of censored observations (i.e., low-level concentrations of POPs with values known to be between zero and 
the laboratory's LOD)[25]. Unadjusted and adjusted censored regression models were used to estimate beta $(\beta)$ coefficients with corresponding $95 \%$ confidence intervals of the relationships between first trimester BMI and lipid-adjusted POPs. The adjusted analyses accounted for the confounding variables as identified above.

The same analytical process was followed to model the association between weight change early in pregnancy and lipid-adjusted POP concentrations.

\section{Results}

\section{Characteristics of study participants}

The baseline characteristics of the MIREC study participants are presented in Table 1. Plasma POP data were collected for 1,983 women at a mean gestational age of 11.6 weeks ( $S D=1.58$ weeks). The majority of participating women were older than 30 years $(69.84 \%)$ and had completed at least an undergraduate degree (62.3\%). Most participants were married or in union (95.3\%), with $>\$ 50,000$ household income (77.8\%). 
Table 1

Characteristics of participants in the Maternal-Infant Research on Environmental Chemicals (MIREC)

Study, $\mathrm{N}=1,983$ pregnant women

\begin{tabular}{|c|c|c|}
\hline & Frequency & Percent \\
\hline \multicolumn{3}{|l|}{ Maternal age group } \\
\hline$<25$ & 139 & 7.01 \\
\hline $25-29$ & 459 & 23.15 \\
\hline $30-34$ & 709 & 35.75 \\
\hline $35+$ & 676 & 34.09 \\
\hline \multicolumn{3}{|l|}{ Maternal education ${ }^{a}$} \\
\hline High school or less & 175 & 8.83 \\
\hline Some college or college degree & 572 & 28.87 \\
\hline Undergraduate degree or higher & 1234 & 62.29 \\
\hline \multicolumn{3}{|l|}{ Marital status } \\
\hline Married or common law & 1889 & 95.26 \\
\hline Other & 94 & 4.74 \\
\hline \multicolumn{3}{|l|}{ Maternal race } \\
\hline Caucasian & 1661 & 83.76 \\
\hline Not Caucasian & 322 & 16.24 \\
\hline \multicolumn{3}{|l|}{ Place of birth } \\
\hline Canada & 1612 & 81.29 \\
\hline Other & 371 & 18.71 \\
\hline \multicolumn{3}{|l|}{ Income } \\
\hline$<\$ 50,000$ & 347 & 17.5 \\
\hline$\$ 50,001-\$ 100,000$ & 786 & 39.64 \\
\hline$>\$ 100,000$ & 757 & 38.17 \\
\hline No answer & 93 & 4.69 \\
\hline
\end{tabular}

aEducation level missing for two participants; ${ }^{b}$ Smoking status missing for 2 participants; Includes women who quit smoking during pregnancy; ${ }^{d}$ Self-reported pre-pregnancy BMI missing for 146 participants; ${ }^{\mathrm{e} F i r s t}$ trimester BMI missing for 71 participants; ${ }^{f}$ Weight change missing for 151 participants. Abbreviations: BMI - Body Mass Index; SD - Standard Deviation 


\section{Smoking status ${ }^{b}$}

Current $^{\mathrm{C}}$

Former

542

27.36

Never

1202

60.68

\section{Pre-pregnancy $\mathrm{BMI}^{\mathrm{d}}$}

Underweight

Normal weight

Overweight

Obese
52

1106

404

275

1013

530

311

58

Class III obese

\section{Weight change category $f$}

Weight loss

72

3.93

Weight neutral or gain

\section{Parity}

0

1

2

$3+$

\section{Fasting status}

No
44.18

40.34

231

11.65

76

3.83

${ }^{a}$ Education level missing for two participants; ${ }^{b}$ Smoking status missing for 2 participants; ${ }^{\text {Includes }}$ women who quit smoking during pregnancy; ${ }^{\mathrm{d}}$ Self-reported pre-pregnancy BMI missing for 146 participants; ${ }^{\mathrm{e}}$ First trimester BMI missing for 71 participants; ${ }^{\text {} W e i g h t ~ c h a n g e ~ m i s s i n g ~ f o r ~} 151$ participants. Abbreviations: BMI - Body Mass Index; SD - Standard Deviation 


\begin{tabular}{|c|c|c|}
\hline & Frequency & Percent \\
\hline Yes & 39 & 2 \\
\hline \multicolumn{3}{|l|}{ Season of blood collection (1st trimester) } \\
\hline Spring & 448 & 22.92 \\
\hline Summer & 464 & 23.73 \\
\hline Fall & 575 & 29.41 \\
\hline Winter & 468 & 23.94 \\
\hline Gestational age at first trimester visit (mean $\pm S D)($ wks) & \multicolumn{2}{|l|}{$11.56(1.58)$} \\
\hline \multicolumn{3}{|c|}{ 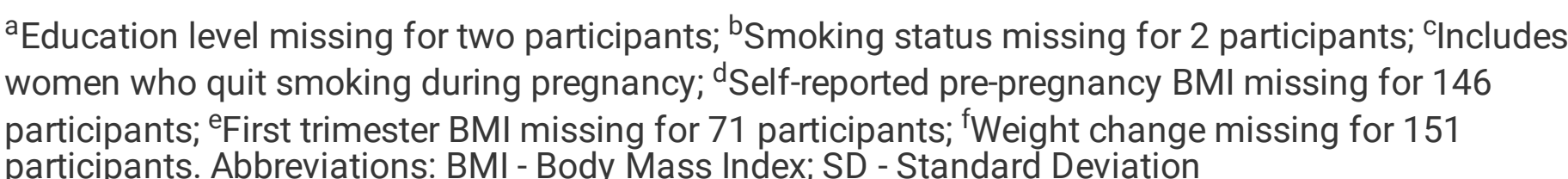 } \\
\hline
\end{tabular}

Self-reported pre-pregnancy BMI was missing for 146 participants (approximately $7.4 \%$ of the sample). About $60 \%$ of the women were classified as being of normal weight based on their pre-pregnancy weight. Close to $15 \%$ of study participants had obesity prior to pregnancy. Of these women, $\%$ has class III obesity. The proportion of women with obesity at the time of the first trimester visit was $19.3 \%$. Of these women. $15.7 \%$ had class III obesity (BMI $\left.\geq 40.0 \mathrm{~kg} / \mathrm{m}^{2}\right)$. Seventy-one participants had missing first trimester BMI measurements. According to our weight change classification, 3.9\% had experienced weight loss during their first trimester.

\section{First trimester POP levels}

The levels of 30 POPs were below their respective LOD in more than $50 \%$ of participants (Table 2). Descriptive statistics are provided for the remaining 11 POPs that had detectable concentrations in more than $50 \%$ of participants. The KM and MLE approaches provided similar descriptive statistics (as shown by the KM medians and geometric means). DDE and Aroclor 1260 exhibited the highest lipid-adjusted geometric mean levels, with concentrations of $56.01 \mu \mathrm{g} / \mathrm{kg}$ serum lipid (95\% Cl: 54.02 - 58.07) and $60.00 \mu \mathrm{g} / \mathrm{kg}$ serum lipid (95\% Cl: 58.11 - 61.95), respectively. Both were detected in more than $95 \%$ of the sample. A maximum DDE concentration of $5306.12 \mu \mathrm{g} / \mathrm{kg}$ serum lipid was noted. PCB170 was the least abundant POP with a concentration of $1.67 \mu \mathrm{g} / \mathrm{kg}$ serum lipid (95\% Cl: $1.58-1.75)$ and was observed in $53.2 \%$ of the MIREC study participants.

Table 2. Distribution of persistent organic pollutant (POP) concentrations ( $\mu \mathrm{g}$ per $\mathrm{kg}$ serum lipid) in maternal blood plasma in 1,983 pregnant women participating in the MIREC Study 


\begin{tabular}{|c|c|c|c|c|c|}
\hline & LOD & $\begin{array}{l}\% \\
<\text { LOD }\end{array}$ & $\begin{array}{l}\text { Maximum } \\
\text { value }\end{array}$ & $\begin{array}{l}\mathrm{KM} \text { median }(95 \% \\
\mathrm{Cl})\end{array}$ & GM (95\% Cl) \\
\hline \multicolumn{6}{|c|}{ Polychlorinated biphenyl (PCB) } \\
\hline РCB28 & 0.05 & 99.74 & 260.00 & NE & NE \\
\hline PCB66 & 0.03 & 99.28 & 28.00 & NE & $\mathrm{NE}$ \\
\hline PCB74 & 0.03 & 96.54 & 18.40 & NE & $\mathrm{NE}$ \\
\hline РCB99 & 0.03 & 97.16 & 12.93 & NE & $\mathrm{NE}$ \\
\hline РCB101 & 0.03 & 99.90 & 12.06 & NE & NE \\
\hline PCB105 & 0.01 & 95.71 & 13.62 & NE & $\mathrm{NE}$ \\
\hline PCB118 & 0.01 & 26.61 & 37.93 & $2.30(2.23,2.38)$ & $2.35(2.29,2.43)$ \\
\hline PCB128 & 0.01 & 99.79 & 5.00 & $\mathrm{NE}$ & $\mathrm{NE}$ \\
\hline PCB138 & 0.01 & 7.03 & 71.66 & $4.01(3.92,4.21)$ & $4.21(4.08,4.37)$ \\
\hline PCB146 & 0.01 & 85.43 & 16.00 & NE & $\mathrm{NE}$ \\
\hline PCB153 & 0.01 & 1.29 & 155.00 & $7.00(6.72,7.29)$ & $7.30(7.06,7.54)$ \\
\hline PCB156 & 0.01 & 79.07 & 17.46 & $\mathrm{NE}$ & $\mathrm{NE}$ \\
\hline PCB163 & 0.01 & 68.11 & 23.81 & $\mathrm{NE}$ & $\mathrm{NE}$ \\
\hline PCB167 & 0.01 & 98.41 & 4.48 & $\mathrm{NE}$ & $\mathrm{NE}$ \\
\hline PCB170 & 0.01 & 46.82 & 71.66 & $1.55(1.29,1.67)$ & $1.67(1.58,1.75)$ \\
\hline РCB178 & 0.01 & 95.71 & 9.02 & $\mathrm{NE}$ & $\mathrm{NE}$ \\
\hline PCB180 & 0.01 & 7.39 & 183.33 & $4.72(4.52,4.90)$ & $4.87(4.70,5.05)$ \\
\hline PCB183 & 0.01 & 91.21 & 23.33 & $\mathrm{NE}$ & $\mathrm{NE}$ \\
\hline PCB187 & 0.01 & 57.21 & 45.00 & NE & $\mathrm{NE}$ \\
\hline PCB194 & 0.01 & 81.17 & 30.00 & NE & $\mathrm{NE}$ \\
\hline РCB203 & 0.01 & 89.35 & 14.16 & NE & $\mathrm{NE}$ \\
\hline РCB201 & 0.01 & 83.72 & 20.00 & NE & $\mathrm{NE}$ \\
\hline РCB206 & 0.01 & 97.67 & 6.61 & NE & $\mathrm{NE}$ \\
\hline Aroclor 1260 & 0.1 & 2.69 & 1183.33 & $57.45(55.29,59.65)$ & $60.00(58.11,61.95)$ \\
\hline \multicolumn{6}{|c|}{ Organochlorines } \\
\hline Beta-HCH & 0.01 & 31.88 & 1108.11 & $2.24(2.16,2.31)$ & $2.32(2.19,2.46)$ \\
\hline Cis-nonachlor & 0.005 & 88.31 & 3.91 & NE & $\mathrm{NE}$ \\
\hline
\end{tabular}




\begin{tabular}{|llllll|}
\hline DDE & 0.09 & 1.03 & 5306.12 & $48.33(46.67,50.00)$ & $56.01(54.02,58.07)$ \\
\hline DDT & 0.05 & 96.28 & 175.44 & NE & NE \\
\hline HCB & 0.04 & 69.54 & 101.66 & NE & NE \\
\hline Mirex & 0.01 & 91.83 & 38.03 & NE & NE \\
\hline Oxychlordane & 0.005 & 7.81 & 17.50 & $2.09(2.00,2.15)$ & $2.00(1.95,2.05)$ \\
\hline Parlar26 & 0.005 & 97.47 & 3.64 & NE & NE \\
\hline Parlar50 & 0.005 & 87.28 & 5.00 & NE & NE \\
\hline Transnonachlor & 0.01 & 15.87 & 34.33 & $2.89(2.69,3.02)$ & $2.90(2.82,2.98)$ \\
\hline Polybrominated diphenyl ethers (PBDE) \& Hexabromobiphenyl (PBB) & \\
\hline PBB153 & 0.02 & 99.48 & 13.61 & NE & NE \\
\hline PBDE100 & 0.02 & 78.46 & 327.27 & NE & NE \\
\hline PBDE153 & 0.02 & 55.60 & 527.27 & NE & NE \\
\hline PBDE28 & 0.03 & 99.07 & 27.14 & NE & NE \\
\hline PBDE33 & 0.03 & 99.90 & 6.28 & NE & NE \\
\hline PBDE47 & 0.03 & 34.28 & 727.27 & $6.97(6.55,7.31)$ & $7.02(6.68,7.38)$ \\
\hline PBDE99 & 0.02 & 80.85 & 169.09 & NE & NE \\
\hline
\end{tabular}

Abbreviations: LOD - Limit of Detection; NE - Not Estimated

\section{POP levels and first trimester BMI}

Figure 1 depicts the lipid-adjusted geometric means and 95\% Cls for the 11 detected POPs. For most POPs, the lipid-adjusted serum concentration was the highest in pregnant women of underweight/normal weight and the lowest in pregnant women with class III obesity. In contrast, PBDE47 concentration increased with first trimester BMI. Beta-HCH was the only POP that did not show variations by first trimester BMI. Kruskal-Wallis tests provided p-values of $<0.0001$ for all POPs, except Beta-HCH (which had a p-value $=0.1195)$.

Similar trends to those depicted in Figure 1 were observed in the unadjusted censored regression models (Table 3). The exponentiated $\beta$ coefficients represent the ratio of the expected geometric means for overweight, class I \& II obese, and class III obese compared with that of underweight/normal weight pregnant women. For instance, for PCB118, the adjusted ratios comparing underweight/normal weight women with overweight, class I \& II obese, and class III obese are $0.96,0.92$, and 0.83 , respectively. These can be interpreted as adjusted geometric means (or concentrations on the log scale) in overweight, class I 
\& II obese, and class III obese pregnant women being approximately $4 \%, 8 \%$, and $17 \%$, respectively, lower than the concentration in underweight/normal weight pregnant women.

The association between first trimester BMI and most POPs remained after adjustment for the main confounders (parity, maternal age, maternal education, maternal smoking status, and mother's place of birth). In both univariate and multivariable models, there was no clear evidence that the Beta-HCH concentrations of overweight, class I \& II obese, and class III obese study participants were significantly different than concentration reported in underweight/normal weight pregnant women. 
Table 3

Unadjusted and adjusted ${ }^{\mathrm{a}}$ associations $[\mathrm{\beta}(95 \% \mathrm{Cl})]$ between first trimester body mass index $(\mathrm{BMI})$ and lipid-adjusted persistent organic pollutant (POP) concentrations detected in at least $50 \%$ of the sample, MIREC Study ${ }^{b}$

\begin{tabular}{|c|c|c|c|c|c|c|}
\hline \multirow[t]{2}{*}{ POP } & \multicolumn{2}{|l|}{ Overweight } & \multicolumn{2}{|c|}{ Class I \& II Obese } & \multicolumn{2}{|c|}{ Class III Obese } \\
\hline & Unadjusted & Adjusted & Unadjusted & Adjusted & Unadjusted & Adjusted \\
\hline \multirow[t]{2}{*}{ PCB118 } & -0.058 & -0.045 & -0.199 & -0.083 & -0.371 & -0.181 \\
\hline & $\begin{array}{l}(-0.127 \\
0.011)\end{array}$ & $\begin{array}{l}(-0.108 \\
0.018)\end{array}$ & $\begin{array}{l}(-0.282 \\
-0.115)\end{array}$ & $\begin{array}{l}(-0.162 \\
-0.003)\end{array}$ & $\begin{array}{l}(-0.548, \\
-0.195)\end{array}$ & $\begin{array}{l}(-0.347 \\
-0.014)\end{array}$ \\
\hline \multirow[t]{2}{*}{ PCB138 } & -0.124 & -0.106 & -0.381 & -0.253 & -0.716 & -0.475 \\
\hline & $\begin{array}{l}(-0.196 \\
-0.052)\end{array}$ & $\begin{array}{l}(-0.169, \\
-0.044)\end{array}$ & $\begin{array}{l}(-0.469, \\
-0.293)\end{array}$ & $\begin{array}{l}(-0.331 \\
-0.174)\end{array}$ & $\begin{array}{l}(-0.900 \\
-0.531)\end{array}$ & $\begin{array}{l}(-0.638 \\
-0.311)\end{array}$ \\
\hline \multirow[t]{2}{*}{ PCB153 } & -0.176 & -0.157 & -0.518 & -0.379 & -0.881 & -0.640 \\
\hline & $\begin{array}{l}(-0.251 \\
-0.102)\end{array}$ & $\begin{array}{l}(-0.219 \\
-0.095)\end{array}$ & $\begin{array}{l}(-0.608 \\
-0.427)\end{array}$ & $\begin{array}{l}(-0.456, \\
-0.303)\end{array}$ & $\begin{array}{l}(-1.068, \\
-0.695)\end{array}$ & $\begin{array}{l}(-0.800 \\
-0.481)\end{array}$ \\
\hline \multirow[t]{2}{*}{ PCB170 } & -0.241 & -0.223 & -0.721 & -0.557 & -1.137 & -0.835 \\
\hline & $\begin{array}{l}(-0.340 \\
-0.142)\end{array}$ & $\begin{array}{l}(-0.309 \\
-0.137)\end{array}$ & $\begin{array}{l}(-0.852 \\
-0.589)\end{array}$ & $\begin{array}{l}(-0.674 \\
-0.439)\end{array}$ & $\begin{array}{l}(-1.452, \\
-0.821)\end{array}$ & $\begin{array}{l}(-1.117 \\
-0.553)\end{array}$ \\
\hline \multirow[t]{2}{*}{ PCB180 } & -0.241 & -0.228 & -0.683 & -0.544 & -1.114 & -0.885 \\
\hline & $\begin{array}{l}(-0.323, \\
-0.158)\end{array}$ & $\begin{array}{l}(-0.295 \\
-0.161)\end{array}$ & $\begin{array}{l}(-0.783, \\
-0.582)\end{array}$ & $\begin{array}{l}(-0.629 \\
-0.459)\end{array}$ & $\begin{array}{l}(-1.328, \\
-0.899)\end{array}$ & $\begin{array}{l}(-1.068, \\
-0.702)\end{array}$ \\
\hline \multirow[t]{2}{*}{ Aroclor 1260} & -0.160 & -0.141 & -0.468 & -0.333 & -0.825 & -0.583 \\
\hline & $\begin{array}{l}(-0.233 \\
-0.087)\end{array}$ & $\begin{array}{l}(-0.202 \\
-0.079)\end{array}$ & $\begin{array}{l}(-0.557 \\
-0.379)\end{array}$ & $\begin{array}{l}(-0.410 \\
-0.256)\end{array}$ & $\begin{array}{l}(-1.009, \\
-0.640)\end{array}$ & $\begin{array}{l}(-0.743, \\
-0.423)\end{array}$ \\
\hline \multirow[t]{2}{*}{ Beta-HCH } & 0.065 & 0.101 & -0.026 & 0.225 & -0.221 & 0.296 \\
\hline & $\begin{array}{l}(-0.070 \\
0.201)\end{array}$ & $\begin{array}{l}(-0.006 \\
0.209)\end{array}$ & $\begin{array}{l}(-0.187 \\
0.138)\end{array}$ & $\begin{array}{l}(-0.092, \\
0.359)\end{array}$ & $\begin{array}{l}(-0.565 \\
0.123)\end{array}$ & $\begin{array}{l}(-0.019 \\
0.574)\end{array}$ \\
\hline \multirow[t]{2}{*}{ DDE } & -0.083 & -0.053 & -0.269 & -0.118 & -0.672 & -0.368 \\
\hline & $\begin{array}{l}(-0.168 \\
0.002)\end{array}$ & $\begin{array}{l}(-0.124 \\
0.018)\end{array}$ & $\begin{array}{l}(-0.372 \\
-0.167)\end{array}$ & $\begin{array}{l}(-0.206 \\
-0.030)\end{array}$ & $\begin{array}{l}(-0.884 \\
-0.459)\end{array}$ & $\begin{array}{l}(-0.549 \\
-0.187)\end{array}$ \\
\hline
\end{tabular}

Statistically significant results are highlighted in bold; ${ }^{a}$ Adjusted for gestational age, maternal age, parity, maternal education, smoking status, and place of birth; bunderweight/Normal weight is the reference category; Abbreviations: $\mathrm{Cl}$ - Confidence Interval, MIREC - Maternal-Infant Research on Environmental Chemicals; PCB - Polychlorinated biphenyl, DDE - Dichlorodiphenyldichloroethylene; PBDE - Polybrominated diphenyl ethers; $\mathrm{HCH}$ - Hexachlorocyclohexane 


\begin{tabular}{|c|c|c|c|c|c|c|}
\hline \multirow[t]{2}{*}{ POP } & \multicolumn{2}{|l|}{ Overweight } & \multicolumn{2}{|c|}{ Class I \& I| Obese } & \multicolumn{2}{|c|}{ Class III Obese } \\
\hline & Unadjusted & Adjusted & Unadjusted & Adjusted & Unadjusted & Adjusted \\
\hline \multirow[t]{2}{*}{ Oxychlordane } & -0.064 & -0.062 & -0.219 & -0.163 & -0.373 & -0.261 \\
\hline & $\begin{array}{l}(-0.122 \\
-0.006)\end{array}$ & $\begin{array}{l}(-0.115 \\
-0.010)\end{array}$ & $\begin{array}{l}(-0.289 \\
-0.149)\end{array}$ & $\begin{array}{l}(-0.228 \\
-0.097)\end{array}$ & $\begin{array}{l}(-0.519 \\
-0.226)\end{array}$ & $\begin{array}{l}(-0.397 \\
-0.125)\end{array}$ \\
\hline \multirow[t]{2}{*}{ Transnonachlor } & -0.064 & -0.066 & -0.258 & -0.191 & -0.403 & -0.282 \\
\hline & $\begin{array}{l}(-0.126 \\
-0.002)\end{array}$ & $\begin{array}{l}(-0.122 \\
-0.010)\end{array}$ & $\begin{array}{l}(-0.334 \\
-0.182)\end{array}$ & $\begin{array}{l}(-0.261 \\
-0.122)\end{array}$ & $\begin{array}{l}(-0.561 \\
-0.244)\end{array}$ & $\begin{array}{l}(-0.428 \\
-0.136)\end{array}$ \\
\hline \multirow[t]{2}{*}{ PBDE47 } & 0.103 & 0.086 & 0.402 & 0.362 & 0.528 & 0.460 \\
\hline & $\begin{array}{l}(-0.011 \\
0.217)\end{array}$ & $\begin{array}{l}(-0.029 \\
0.201)\end{array}$ & $\begin{array}{l}(0.266 \\
0.537)\end{array}$ & $\begin{array}{l}(0.222, \\
0.502)\end{array}$ & $\begin{array}{l}(0.254 \\
0.802)\end{array}$ & $\begin{array}{l}(0.177, \\
0.743)\end{array}$ \\
\hline \multicolumn{7}{|c|}{$\begin{array}{l}\text { Statistically significant results are highlighted in bold; }{ }^{\circ} \text { Adjusted for gestational age, maternal age, } \\
\text { parity, maternal education, smoking status, and place of birth; bUnderweight/Normal weight is the } \\
\text { reference category; Abbreviations: CI - Confidence Interval, MIREC - Maternal-Infant Research on } \\
\text { Environmental Chemicals; PCB - Polychlorinated biphenyl, DDE - Dichlorodiphenyldichloroethylene; } \\
\text { PBDE - Polybrominated diphenyl ethers; HCH - Hexachlorocyclohexane }\end{array}$} \\
\hline
\end{tabular}

\section{POP levels and early pregnancy weight change}

The unadjusted concentrations of most analyzed POPs tended to be slightly higher in women who were classified in the weight neutral/gain group (Figure 2). Kruskal-Wallis tests returned p-values greater than 0.05 for all POPs (values not shown).

In the unadjusted analyses, the plasma concentrations of POPs were not significantly different between women who lost weight and those who gained weight or remained weight neutral during their first trimester (Table 4). After controlling for the confounders (parity, maternal age, maternal education, maternal smoking status, mother's place of birth and pre-pregnancy BMI), the $\beta$ coefficients for the majority of POPs were positive, suggesting that, compared to the weight neutral/gain category, the concentrations of circulating POPs were higher among women who had a first trimester weight loss of at least $2 \%$ of their total body weight. Still, none of the findings were statistically significant, as depicted by the $95 \%$ confidence intervals including zero. 
Table 4

Unadjusted and adjusted ${ }^{\mathrm{a}}$ associations $[\mathrm{B}(95 \% \mathrm{Cl})]$ between first trimester weight change $(\mathrm{BMI})$ and lipidadjusted persistent organic pollutants (POPs) concentrations detected in at least $50 \%$ of the samples, MIREC Study ${ }^{b}$

\begin{tabular}{|c|c|c|}
\hline & Weight loss & \\
\hline & Unadjusted & Adjusted \\
\hline \multirow[t]{2}{*}{ PCB118 } & 0.075 & 0.144 \\
\hline & $(-0.080,0.230)$ & $(-0.003,0.291)$ \\
\hline \multirow[t]{2}{*}{ PCB138 } & -0.002 & 0.146 \\
\hline & $(-0.168,0.164)$ & $(0.0003,0.292)$ \\
\hline \multirow[t]{2}{*}{ PCB153 } & -0.052 & 0.143 \\
\hline & $(-0.226,0.121)$ & $(-0.001,0.288)$ \\
\hline \multirow[t]{2}{*}{ PCB170 } & -0.152 & 0.112 \\
\hline & $(-0.391,0.087)$ & $(-0.098,0.323)$ \\
\hline \multirow[t]{2}{*}{ PCB180 } & -0.148 & 0.134 \\
\hline & $(-0.343,0.045)$ & $(-0.024,0.293)$ \\
\hline \multirow[t]{2}{*}{ Aroclor 1260} & -0.028 & 0.148 \\
\hline & $(-0.198,0.141)$ & $(0.004,0.292)$ \\
\hline \multirow[t]{2}{*}{ Beta-HCH } & -0.008 & 0.019 \\
\hline & $(-0.314,0.297)$ & $(-0.226,0.254)$ \\
\hline \multirow[t]{2}{*}{ DDE } & 0.039 & 0.123 \\
\hline & $(-0.152,0.229)$ & $(-0.038,0.285)$ \\
\hline \multirow[t]{2}{*}{ Oxychlordane } & -0.056 & 0.031 \\
\hline & $(-0.187,0.074)$ & $(-0.091,0.152)$ \\
\hline \multirow[t]{2}{*}{ Transnonachlor } & -0.112 & 0.015 \\
\hline & $(-0.254,0.029)$ & $(-0.115,0.144)$ \\
\hline
\end{tabular}

${ }^{a}$ Adjusted for gestational age, maternal age, parity, pre-pregnancy BMI, maternal education, smoking status, and place of birth; ${ }^{b}$ Weight neutral/gain is the reference category; Abbreviations: $\mathrm{Cl}-$ Confidence Interval, MIREC - Maternal-Infant Research on Environmental Chemicals; PCB Polychlorinated biphenyl, DDE - Dichlorodiphenyldichloroethylene; PBDE - Polybrominated diphenyl ethers; $\mathrm{HCH}$ - Hexachlorocyclohexane 


\section{Weight loss}

PBDE47

0.172

$(-0.083,0.426)$
$-0.008$

$(-0.273,0.258)$

${ }^{a}$ Adjusted for gestational age, maternal age, parity, pre-pregnancy BMI, maternal education, smoking status, and place of birth; ${ }^{b}$ Weight neutral/gain is the reference category; Abbreviations: $\mathrm{Cl}-$ Confidence Interval, MIREC - Maternal-Infant Research on Environmental Chemicals; PCB Polychlorinated biphenyl, DDE - Dichlorodiphenyldichloroethylene; PBDE - Polybrominated diphenyl ethers; $\mathrm{HCH}$ - Hexachlorocyclohexane

\section{Discussion}

\section{Summary of findings and clinical significance of the findings}

Thirty of the 41 POPs collected in the MIREC Study were below their respective LOD in over $50 \%$ of the participants. Population-based national surveys from Canada[28] and the United States[29], as well as observational studies from Canada[19], Mexico[19], Greece[30], and Lebanon[16] suggest relatively low levels of exposure to circulating POPs in general and obstetrical populations. As described previously, women participating in the MIREC study had lower plasma concentrations of Aroclor 1260 and DDE than women of equivalent age (20 to 39 years of age) participating in the 2012 - 2013 Canadian Health Measures Survey report on Human Biomonitoring of Environmental Chemicals in Canada[28] and those surveyed in the Fourth National Report on Human Exposure to Environmental Chemicals of the Centre for Disease Control[29]. The most dominant compounds in the MIREC study were Aroclor 1260 and DDE. DDE was also the dominant contaminant in a number of other maternal-newborn cohorts[19, 31, 32].

This study underscores the original findings from the MIREC study cohort that environmental and chemical contaminants are quantifiable in the plasma of Canadian pregnant women[23] and provides further insight into the relative body burden of POPs among pregnant women across categories of first trimester $\mathrm{BMI}$ and early pregnancy weight changes. We expanded on previous findings to demonstrate that first trimester plasma levels of multiple POPs differed significantly across categories of first trimester BMI. Oxychlordane, Aroclor 1260, transnonachlor, PCB 170, 180, 118, 138 and, 153, and DDE plasma levels were negatively associated with first-trimester BMI. Put simply, the highest POP concentrations were found in the underweight and normal weight women, and the lowest in women with class III obesity. Inverse relationships between circulating concentrations of POPs and BMI in pregnancy have been previously reported in other studies[15, 18,33]. Results are also in line with other Canadian studies that looked specifically at pre-pregnancy BMI[32]. The inverse relationships noted for most POPs can be explained by the known biochemistry of POPs: Lipophilic compounds that tend to be stored in the adipose tissues rather than in circulation[18]. In contrast to previous findings[18, 23, 34], we did not detect a positive relationship between Beta- $\mathrm{HCH}$ concentrations and maternal $\mathrm{BMI}$, although our ability to study 
this association may have been limited by the low detection rate of Beta-HCH compound in our samples (approximately 32\%). Interestingly, PBDE47 was the only POP that had a positive relationship with BMI. Similar findings were noted by Fisher et al. for the association between pre-pregnancy BMI and POP serum concentrations[23]. Minimal evidence is available on this POP's association with maternal BMI. As demonstrated by research studies of pregnant women from Denmark and Mexico[18, 19], it is a POP with a low detection level. Studies from North America, where concentrations of PBDEs are known to be greater than in other parts of the world, have, however, found concentrations of various PBDE to increase with pre-pregnancy BMI and have suggested that PBDEs may have an obesogenic effect and accelerate the proliferation of adipose cells $[25,35]$. It can also be noted that, compared to PCBs, PBDEs represent a 'younger' class of POPs and could therefore have a different distribution in the body than PCBs. Given the known effects of obesity on maternal and fetal health, additional research is needed to clarify the mechanisms linking PBDEs with obesity.

Weight changes are expected in pregnancy. A small number of women will lose weight or remain weight neutral during pregnancy. This may be achieved with or without intention. Many will become motivated to make lifestyle changes to improve overall health, such as consuming a healthier diet or engaging in more physical activity. Others may be affected with significant nausea and vomiting or worsening of preexisting medical conditions. Our study observed slightly higher POP concentrations (after controlling for confounders) in pregnant women who lost weight compared to those who gained weight or remained weight neutral during the first trimester. On the premise that individuals with a higher body fat composition would have a higher POP storage capacity, weight loss, particularly among overweight and obese individuals, is typically associated with a redistribution of POPs from the adipose tissues into the bloodstream $[8,10,35]$. POPs have been shown to cross the placenta, and intrauterine exposure has been associated with low birth weight and prematurity[20, 21, 30]. Furthermore, alteration of immune system development, endocrine disruption and abnormal growth and development has been observed in children with early exposure to POPs[12, 14, 22, 30]. The release of POPs into circulation during pregnancy in women who lose weight, regardless of etiology, could become of clinical importance as more evidence becomes available.

While it was beyond the current project scope, evaluation of maternal POPs serum concentration relative to maternal weight variations between pregnancy trimesters in women who participated in the MIREC project and other prospective cohorts will be important to carry out in future studies. POPs have previously been clearly shown to interfere with biologic systems. The first step in understanding their clinical significance in pregnancy is describing the burden of POPs and the factors that contribute to increased levels. If weight change in pregnancy (most likely weight loss) results in increased levels of circulating POPs, then study of the potential harmful impact on the mother, developing fetus, and placenta should be considered. If proven, patients and health care providers could benefit from counseling around strategies to optimize a healthy GWG and about the possible risks of increased POPs exposure in pregnancy, whenever possible.

\section{Strengths and limitations}


The strengths of this study include the use of a well-characterized cohort and concurrent sampling of first trimester $\mathrm{BMI}$ and maternal plasma for POP analysis. We were also able to consider a large number of POPs in our analysis. Additionally, we included key confounding variables in our censored regression models. Still, other important variables capturing dietary habits of the participants (e.g., fish consumption) were not considered in our analyses; this should be noted as a limitation of our study. Exclusion of key confounders may have resulted in residual confounding and biased our estimation of the associations of first trimester BMI and early pregnancy weight change with POPs. Furthermore, this analysis relied on the MIREC Study's baseline data, which were cross-sectional, thus preventing to clearly define the direction of some of the associations. As reported in some prospective cohort studies, POPs may lead to low gestational weight change throughout pregnancy[12, 14, 22, 30, 37]. Other studies conducted in Canada have also shown that certain environmental chemicals may be associated with increased GWG[38].

More studies investigating the mechanisms linking maternal weight changes and POPs are required. Our findings are limited by a small sample size ( $N=72$ pregnant women experienced weight loss in the first trimester), possible inaccuracy of self-reported pre-pregnancy weight, and a short time interval between pre-pregnancy and first-trimester weight measurements. Self-reported pre-pregnancy weight may have limited the accuracy of our weight change calculations. However, it is important to note that self-reported maternal weight is commonly used in clinical practice to calculated pre-pregnancy BMI. Studies have shown a high correlation between self-reported and measured pre-pregnancy weight[38]. When discrepancies were observed between the two measurements, this generally did not misclassify BMI class[38]. Furthermore, maternal body composition may be stable during the first trimester POPs may, in fact, be more strongly related to weight changes over longer time periods than that observed during pregnancy [36].

In addition, results of this study cannot be generalized to all populations. Most participants were recruited from southern urban settings. Similarly, women from the MIREC study were older, less likely to smoke, and more likely to be married, and to be educated than the general Canadian population giving birth in 2009[24]. Lastly, while the majority of the POPs considered in our study (30/41) had levels below their respective LODs in more than $50 \%$ of samples, we attempted to address this by limiting our analyses to those POPs with less than $50 \%$ of samples under the LOD.

\section{Conclusions}

In conclusion, first trimester plasma concentrations of POPs are negatively associated with maternal first trimester BMI, implying that women with obesity may indeed have POPs sequestrated in adipose tissues. Circulating POPs concentrations were higher in women exhibiting weight loss compared to those with gain or weight neutrality in the first trimester of pregnancy. This relationship and its underlying mechanisms remain to be confirmed in studies with larger samples of pregnant women and longer time intervals between weight measurements. Additional research is warranted to further evaluate the dynamic interplay between gestational weight change and circulating POP concentrations across the continuum 
of pregnancy. Such work would be beneficial for informing gestational weight gain recommendations, particularly for pregnant women with obesity, who potentially may have a high body burden of POPs.

\section{Abbreviations}

Beta-HCH: Beta- Hexachlorocyclohexane

BMI: Body Mass Index

DDE: Dichlorodiphenyldichloroethylene

DDT: Dichlorodiphenyltrichloroethane

GWG: Gestational Weight Gain

LOD: Limit of Detection

MIREC: Maternal-Infant Research on Environmental Chemicals

PBB: Polybrominated biphenyl

PBDE: Polybrominated diphenyl ether

PCB: Polychlorinated biphenyl

POP: Persistent Organic Pollutant

\section{Declarations}

\section{Ethics approval and consent to participate}

This study was approved by the Ottawa Health Science Network Research Ethics Board (Approval number: 20140736). Applications were also filled to access the MIREC Study data. The authors confirm that all methods were performed in accordance with relevant guidelines and regulations. All participating women provided informed consent.

\section{Consent for publication}

Not applicable

\section{Availability of data and materials}

Permission to use the data that support the findings of this study can be obtained from the MIREC Study Group upon reasonable request. 
The authors declare no conflict of interest.

\section{Funding}

Canadian Institutes of Health Research (CIHR), Grant/Award Number: MFM-146444.

\section{Authors' contributions}

LMG conceived the project. $L M G, E B, M L$, and MOO developed the protocol and the analytical plan. MOO performed all data preparation and analyses, with $L M G, M L, R F$, and $A D H$ providing guidance on the statistical approach. ML and MOO wrote the manuscript with input from all authors. All authors interpreted the findings and contributed to the writing and completion of the manuscript. All authors read and approved the final version of the manuscript.

\section{Acknowledgments}

The MIREC Biobank is acknowledged as the source of data used in this project. The authors thank the participants and staff of the MIREC study, along with the MIREC Site Co-Investigators. We acknowledge Jillian Ashley-Martin, who reviewed this manuscript on behalf of the MIREC Study Group. We also acknowledge all members of the MIREC Study Group. The MIREC study group consists of: TE Arbuckle and WD Fraser (MIREC Study Principal Investigators) and D Jin, T Rawn, B Dabeka, M Fisher, S Brooks, XL Cao, K Cockell, A Becalski, R Vincent, M Villeneuve, Z Wang, N Ratnayake (retired), G Bondy, M D'Amour, I Massarelli, A MacFarlane, N Hidiroglou (deceased), P Kumarathasan, M Legrand, S Tittlemier, D Avard (retired), JP Weber (retired), R Platt, H Weiler, A Ettinger, G Mitchell, ZC Luo, A LeBlanc, P Julien, W Foster, G Smith, M Sermer, G Ross, P Fredette, M Lawson, R Shear, W Fraser, M Helewa, S Taback, D Hemmings, J Wang, P von Dadelszen and B Lanphear (all MIREC Study Co-investigators). The MIREC study was supported by Health Canada, the Ontario Ministry of the Environment, and the Canadian Institutes of Health Research.

\section{References}

[1] Crane JMG, Murphy P, Burrage L, et al. Maternal and Perinatal Outcomes of Extreme Obesity in Pregnancy. Journal of Obstetrics and Gynaecology Canada 2013; 35: 606-611.

[2] Arendas K, Qiu Q, Gruslin A. Obesity in Pregnancy: Pre-Conceptional to Postpartum Consequences. Journal of Obstetrics and Gynaecology Canada 2008; 30: 477-488.

[3] Block SR, Watkins SM, Salemi JL, et al. Maternal pre-pregnancy body mass index and risk of selected birth defects: Evidence of a dose-response relationship. Paediatric and Perinatal Epidemiology 2013; 27: $521-531$.

[4] Voerman E, Santos S, Inskip H, et al. Association of Gestational Weight Gain With Adverse Maternal and Infant Outcomes. JAMA 2019; 321: 1702-1715. 
[5] Maxwell C, Gaudet L, Cassir G, et al. Guideline No. 391-Pregnancy and Maternal Obesity Part 1: Preconception and Prenatal Care. Journal of Obstetrics and Gynaecology Canada 2019; 41: 1623-1640.

[6] Health Canada. Prenatal Nutrition Guidelines for Health Professionals: Gestational Weight Gain, https://www.canada.ca/content/dam/hc-sc/migration/hc-sc/fn-

an/alt_formats/pdf/nutrition/prenatal/ewba-mbsa-eng.pdf (2010, accessed September 14, 2021).

[7] Woolcott C, Dodds L, Ashley-Martin J, et al. Distribution of pregnancy-related weight measures. Canadian Family Physician 2016; 62: e400-e406.

[8] Malarvannan G, van Hoorenbeeck K, Deguchtenaere A, et al. Dynamics of persistent organic pollutants in obese adolescents during weight loss. Environment International 2018; 110: 80-87.

[9] Pestana D, Faria G, Sá C, et al. Persistent organic pollutant levels in human visceral and subcutaneous adipose tissue in obese individuals-Depot differences and dysmetabolism implications. Environmental Research 2014; 133: 170-177.

[10] Lim JS, Son HK, Park SK, et al. Inverse associations between long-term weight change and serum concentrations of persistent organic pollutants. International Journal of Obesity 2011; 35: 744-747.

[11] Hagen PE, Walls MP. "The Stockholm Convention On Persistent Organic Pollutants ". 2005.

[12] Ruzzin J. Public health concern behind the exposure to persistent organic pollutants and the risk of metabolic diseases. BMC Public Health; 12. Epub ahead of print 2012. DOI: 10.1186/1471-2458-12-298.

[13] Lallas PL. The Stockholm Convention on Persistent Organic Pollutants. American Journal of International Law 2001; 95: 692-708.

[14] World Health Organization. Persistent Organic Pollutants: Impact on Child Health, https://apps.who.int/iris/bitstream/handle/10665/44525/9789241501101_eng.pdf? sequence=1\&isAllowed=y (2010, accessed July 20, 2021).

[15] Wang RY, Jain RB, Wolkin AF, et al. Serum Concentrations of Selected Persistent Organic Pollutants in a Sample of Pregnant Females and Changes in Their Concentrations during Gestation. Environmental Health Perspectives 2009; 117: 1244.

[16] Helou K, Matta J, Harmouche-Karaki M, et al. Maternal and cord serum levels of polychlorinated biphenyls (PCBs) and organochlorine pesticides (OCPs) among Lebanese pregnant women and predictors of exposure. Chemosphere 2021; 266: 129211.

[17] Vizcaino E, Grimalt JO, Glomstad B, et al. Gestational Weight Gain and Exposure of Newborns to Persistent Organic Pollutants. Environmental Health Perspectives 2014; 122: 873. 
[18] Bjerregaard-Olesen C, Long M, Ghisari M, et al. Temporal trends of lipophilic persistent organic pollutants in serum from Danish nulliparous pregnant women 2011-2013. Environmental Science and Pollution Research 2017; 24: 16592-16603.

[19] Adlard B, Davis K, Liang CL, et al. Persistent organic pollutants (POPs) and metals in primiparous women: A comparison from Canada and Mexico. Science of the Total Environment 2014; 500-501: 302313.

[20] Longnecker MP, Klebanoff MA, Zhou H, et al. Association between maternal serum concentration of the DDT metabolite DDE and preterm and small-for-gestational-age babies at birth. Lancet $2001 ; 358$ : 110-114.

[21] Nieuwenhuijsen MJ, Dadvand P, Grellier J, et al. Environmental risk factors of pregnancy outcomes: a summary of recent meta-analyses of epidemiological studies. Environmental health: a global access science source, 12. Epub ahead of print 2013. DOI: 10.1186/1476-069X-12-6.

[22] World Health Organization. Principles for evaluating health risks in children associated with exposure to chemicals., https://apps.who.int/iris/handle/10665/43604 (2006, accessed July 20, 2021).

[23] Fisher M, Arbuckle TE, Liang CL, et al. Concentrations of persistent organic pollutants in maternal and cord blood from the maternal-infant research on environmental chemicals (MIREC) cohort study. Environmental Health: A Global Access Science Source, 15. Epub ahead of print May 4, 2016. DOI: 10.1186/s12940-016-0143-y.

[24] Arbuckle TE, Fraser WD, Fisher M, et al. Cohort profile: The maternal-infant research on environmental chemicals research platform. Paediatric and Perinatal Epidemiology 2013; 27: 415-425.

[25] Mehta SS, Applebaum KM, James-Todd T, et al. Associations between sociodemographic characteristics and exposures to PBDEs, OH-PBDEs, PCBs, and PFASs in a diverse, overweight population of pregnant women. Journal of exposure science \& environmental epidemiology 2020; 30: 42.

[26] Williamson EJ, Aitken Z, Lawrie J, et al. Introduction to causal diagrams for confounder selection. Respirology 2014; 19: 303-311.

[27] Helsel DR. Statistics for Censored Environmental Data Using Minitab® and R. Second Edition. John Wiley and Sons. Epub ahead of print December 7, 2011. DOI: 10.1002/9781118162729.

[28] Health Canada. Third Report on Human Biomonitoring of Environmental Chemicals in Canada, https://www.canada.ca/content/dam/hc-sc/migration/hc-sc/ewhsemt/alt_formats/pdf/pubs/contaminants/chms-ecms-cycle3/chms-ecms-cycle3-eng.pdf (2015, accessed July 20, 2021).

[29] Centre for Disease Control and Prevention (CDC). Fourth National Report on Human Exposure to Environmental Chemicals, https://www.cdc.gov/nchs/nhanes/. (2021, accessed July 20, 2021). 
[30] Vafeiadi M, Georgiou V, Chalkiadaki G, et al. Association of prenatal exposure to persistent organic pollutants with obesity and cardiometabolic traits in early childhood: The rhea mother-child cohort (Crete, Greece). Environmental Health Perspectives 2015; 123: 1015-1021.

[31] Adetona O, Horton K, Sjodin A, et al. Concentrations of select persistent organic pollutants across pregnancy trimesters in maternal and in cord serum in Trujillo, Peru. Chemosphere 2013; 91: 1426-1433.

[32] Foster WG, Cheung AP, Davis K, et al. Circulating metals and persistent organic pollutant concentrations in Canadian and non-Canadian born primiparous women from five Canadian centres: Results of a pilot biomonitoring study. Science of the Total Environment 2012; 435-436: 326-336.

[33] Glynn A, Aune M, Darnerud PO, et al. Determinants of serum concentrations of organochlorine compounds in Swedish pregnant women: a cross-sectional study. Environmental Health 2007; 6: 2.

[34] Dirinck E, Jorens PG, Covaci A, et al. Obesity and Persistent Organic Pollutants: Possible Obesogenic Effect of Organochlorine Pesticides and Polychlorinated Biphenyls. obesity, 19. Epub ahead of print 2011. DOI: $10.1038 /$ oby.2010.133.

[35] Vuong AM, Braun JM, Sjödin A, et al. Prenatal Polybrominated Diphenyl Ether Exposure and Body Mass Index in Children Up To 8 Years of Age. Environmental Health Perspectives 2016; 124: 1891.

[36] Jansen A, Lyche JL, Polder A, et al. Increased blood levels of persistent organic pollutants (POP) in obese individuals after weight loss-A review. Journal of Toxicology and Environmental Health - Part B: Critical Reviews 2017; 20: 22-37.

[37] Jaacks LM, Barr DB, Sundaram R, et al. Pre-Pregnancy Maternal Exposure to Persistent Organic Pollutants and Gestational Weight Gain: A Prospective Cohort Study. International Journal of Environmental Research and Public Health 2016; 13: 905.

[38] Ashley-Martin J, Dodds L, Arbuckle TE, et al. Maternal and Neonatal Levels of Perfluoroalkyl Substances in Relation to Gestational Weight Gain. International Journal of Environmental Research and Public Health 2016; 13: 146.

[39] I H, AK C, M M, et al. The accuracy of self-reported pregnancy-related weight: a systematic review. Obesity reviews: an official journal of the International Association for the Study of Obesity 2017; 18: 350-369.

\section{Figures}



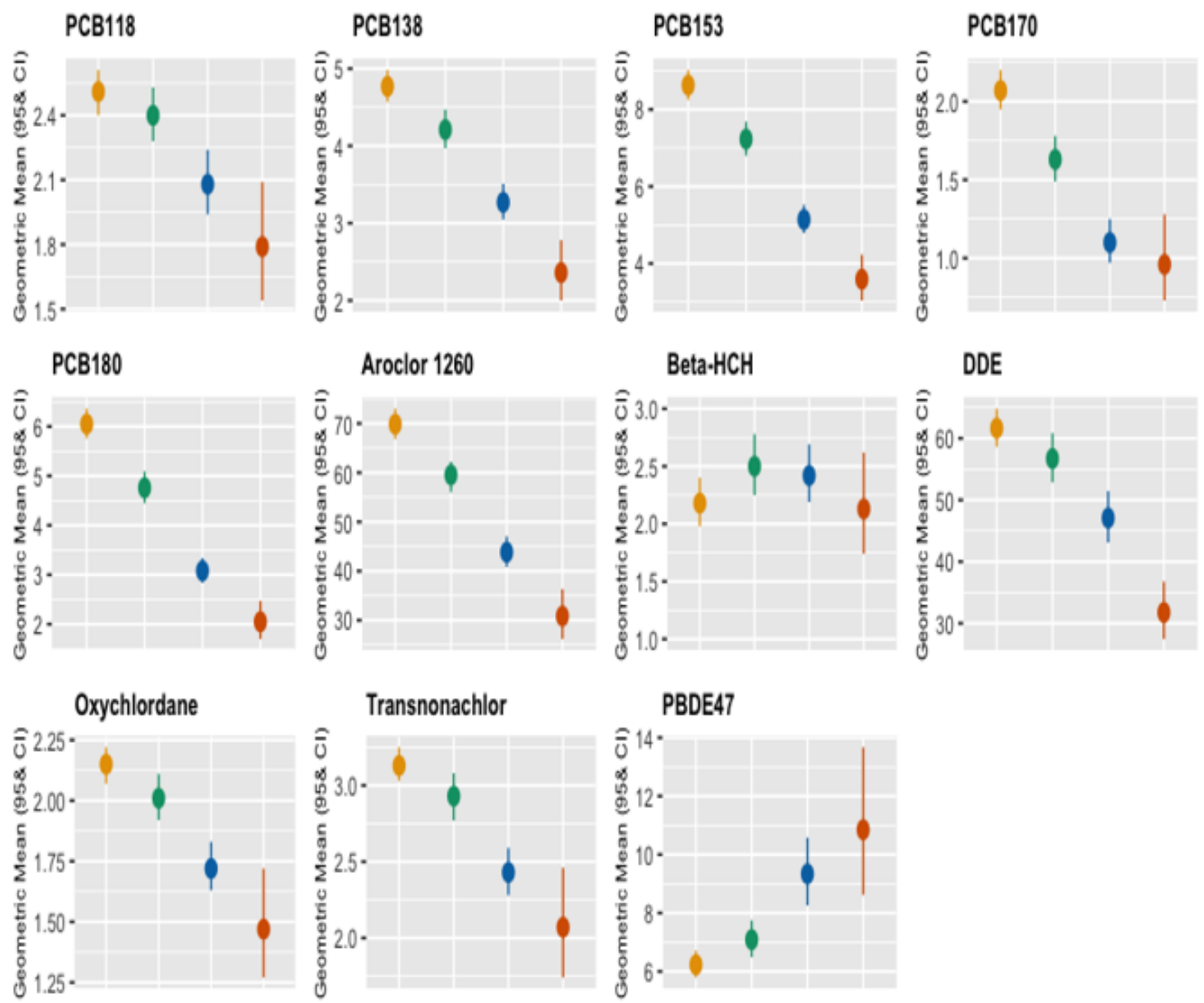

First trimester BMI $\phi$ UnderweightNormal weight $\phi$ Overweight $\phi$ Class $1 \&$ II Obese $\phi$ Class III Obese

\section{Figure 1}

Geometric means and 95\% confidence limits of eleven POP concentrations by first trimester BMI category, $\mathrm{N}=1,912$ pregnant women, MIREC Study 

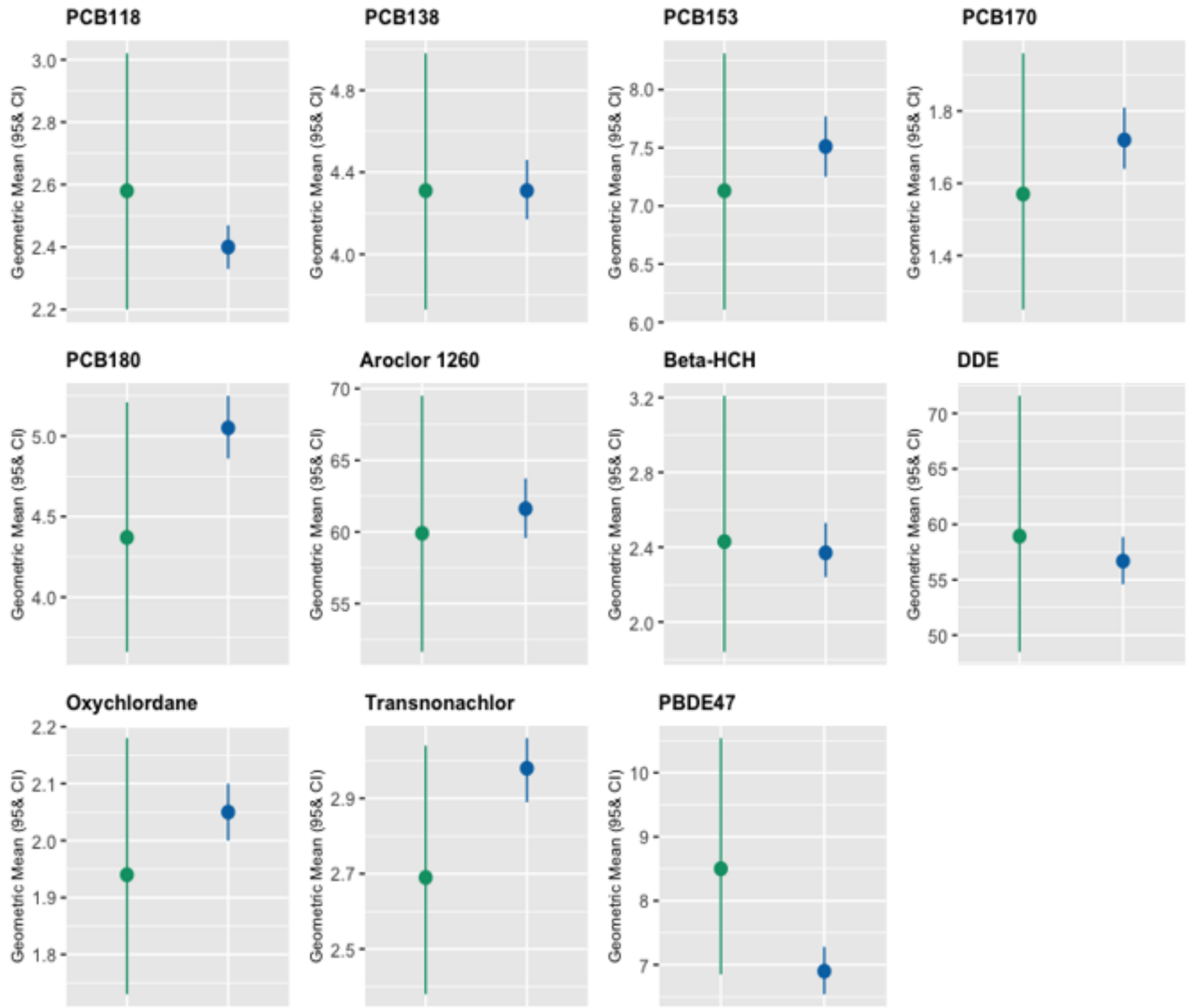

Weightchange $\phi$ Weight loss

Weight neutral/gain

\section{Figure 2}

Geometric means and $95 \%$ confidence limits of eleven POP concentrations by early gestational weight change category, $\mathrm{N}=1,832$ pregnant women, MIREC Study 